ГORIGINAL ARTICLE-

Volume 13 Issue 22021

DOI: 10.21315/eimj2021.13.2.4

ARTICLE INFO

Received: 28-11-2020

Accepted: 10-04-2021

Online: 30-06-2021

\section{Computer-Based Simulation by Emergency Medicine Resident-Educator for Medical Students during the COVID-19 Pandemic}

\author{
Ho Shu Fang ${ }^{1}$, Tan Ming Jing Elizabeth' ${ }^{1}$, Fatimah Lateef ${ }^{1,2,3,4}$ \\ ${ }^{1}$ Department of Emergency Medicine, Singapore General Hospital, \\ SINGAPORE \\ ${ }^{2}$ Yong Loo Lin School of Medicine, National University of Singapore, \\ SINGAPORE \\ ${ }^{3}$ Lee Kong Chian Medical School, Nanyang Technological University, \\ SINGAPORE \\ ${ }^{4}$ Duke-NUS Graduate Medical School, SINGAPORE
}

To cite this article: Ho SF, Tan MJE, Lateef F. Computer-based simulation by emergency medicine resident-educator for medical students during the COVID-19 pandemic. Education in Medicine Journal. 2021;13(2):41-53. https://doi.org/10.21315/eimj2021.13.2.4

To link to this article: https://doi.org/10.21315/eimj2021.13.2.4

\title{
ABSTRACT
}

Today, residents in all disciplines are expected to be involved in not just educating themselves but in the education of others and peers as well. They are involved in a wide spectrum of teaching and instruction techniques such as case presentations, lectures, practical hands-on teaching, bedside clinical tutorials, informal discussions and simulation-based training. Simulation-based teaching has been playing an increasingly important role in both residency training as well as medical school curricula. In particular, it appeals to adult learners as it very task-driven and task-oriented, it allows for constant active engagement during role-playing in simulated scenarios and enables repetitive practice until a certain level of mastery or competency is achieved. The SingHealth residents training in emergency medicine have been collaborating with and engaging medical students from the Duke-NUS Graduate Medical School, as the two entities for a common Academic Medical Center. They share many collaborative projects and activities, research as well as educational training programmes. However, with the recent COVID-19 pandemic, both face-to-face medical teaching as well as simulation-based teaching proved to be challenging. One alternative is to move these teaching collaborations and programmes onto the online platform. This study describes the experience of emergency medicine resident-educators who conducted emergency medicine computer-based simulations (CBS) in collaboration with a group of medical students from the Duke-NUS Emergency Medicine Student Interest Group during the COVID-19 pandemic.

Keywords: Computer-based simulation, Resident-educator, Simulation, Debriefing, Medical students, Psychological safety

Fatimah Lateef, Department of Emergency Medicine, Singapore General Hospital, Outram Road, 1 Hospital Drive, 169608 Singapore

Email: fatimah.abd.lateef@singhealth.com.sg 


\section{INTRODUCTION}

Residents in training have to fulfill multiple roles which span some five to six years. These include their roles as caregiver in managing patients, researcher, educator, mentor and supervisor as well as befriender. In recent years, there has been increasing recognition of the resident's role as an educator to medical students and other residents or peers. In order to prepare residents for a career in academic medicine as a clinician-educator, teaching experience has increasingly become a key component of a resident's portfolio (1-3). The SingHealth Emergency Medicine Residency Program's (SHEMRP), faculty and residents, maintain a close relationship with the Duke-NUS Medical School's Emergency Medicine Student Interest Group (Emed SIG). This relationship has bloomed multiple synergistic teaching and mentorship collaborations, of which simulation-based teaching was the best received and most popular, amongst the medical students. The residents contribute to medical students' education via lectures, small and large group instruction, supervision, mentoring, casual conversation, discussions and even befriending (2). They tend to teach differently from faculty; usually on the job when students are embedded with them, complementary to faculty teaching sessions, helping in deconstruction of complex concepts and procedures to enhance student understanding as well as sharing, based on their own experiences when they themselves had to go through the same challenges. As they educate, these residents learn more, increase their enthusiasm and may even increase their own job satisfaction (3-5). At the same time, they grow to understand their role as clinician-educators better and with greater depth.

Residents are involved in a wide spectrum of teaching and instruction techniques. Some of these are case presentations, lectures, practical hands-on teaching, bedside clinical tutorials, informal discussions and simulation-based training. Simulation-based teaching has been playing an increasingly important role in both residency training as well as medical school curricula. In particular, it appeals to adult learners as it is very task-driven and task-oriented, it allows for constant active engagement during role-playing in simulated scenarios as well as enables repetitive practice until a certain level of mastery or competency is achieved. In this context, SHEMRP has been collaborating with and engaging medical students through both high and low fidelity simulation-based learning. However, with the recent COVID-19 pandemic, both face-to-face medical teaching as well as simulation-based teaching proved to be a challenge. The natural solution is to move these teaching collaborations and programmes online (6-8).

This study describes the experience of emergency medicine resident-educator volunteers who conducted emergency medicine computer-based simulations (CBS) in collaboration with a group of medical students from the Emed SIG during the COVID-19 pandemic. This initiative came about and was conceptualised out of the need to conduct educational sessions despite all the restrictions on face-toface training, due to the pandemic. It represents a descriptive sharing of the programme which started during the COVID-19 outbreak. This programme is expected to continue into the new norm, post-COVID-19, where there are plans to supplement it with actual face-to-face simulation sessions in the simulation laboratory (9). The resident-educators also plan to pursue this further as a longitudinal research project, following the positive feedback from this pilot implementation.

\section{METHODOLOGY}

\section{Establishing Learning Objectives}

Participation in the CBS session was entirely on a voluntary basis. Both the residents and medical students did this in their own time, 
outside of any formal curriculum time. The main focus was to continue with education and the imparting of knowledge, despite the restrictions and distancing measures imposed by the pandemic. In view that this was an initiative in education by residenteducators for the emergency medicine simulation interest group students, and it was not planned as a research proposal or study, Institutional Review Board approval was not indicated.

Learning objectives were clearly established following discussions between both parties. The learning objectives for the residenteducator were set as follows:

a. To conceptualise and design emergency medicine based scenarios suitable for medical students who were in their pre-clinical and early clinical years;

b. To conduct the CBS for the medical students; and

c. To conduct debriefing after the simulation sessions.

The above objectives would also align with the requirements for emergency medicine residents to fulfil for their education portfolio, the objectives of which include their exposure to a wide variety of educational methodologies, in a variety of contexts as well as get used to the various spectrum of learners.

The medical students who signed up with the Emed SIG were individually surveyed via an online form at the pre-participation stage. They were asked for:

a. Their level of training and prior clinical exposure;

b. Their reasons for signing up for the CBS sessions; and

c. Their personal top three learning objectives.

Pre-determined learning objectives were also provided for the students to select. These included: interest in emergency medicine; gaining greater exposure to emergency case management; to experience the dynamic nature of the work in the emergency department; to challenge oneself with case handling under pressure; and to learn more about simulation-based education. They were also asked about their perceived greatest fear when helping the medical team in managing critically ill patients in the emergency department.

\section{Preparing the Resident-Educator}

None of the resident-educator have had formal training in designing and conducting simulation-based learning or computerbased simulation. They have however, been through multiple simulation-based learning sessions during their residency training.

In order to assist the resident-educator in achieving their learning objectives, they had a two-hour introductory session to CBS conducted by an experienced faculty from the medical school. They were introduced to the concept of CBS and how it was to be carried out. Pre-recorded segments of CBS by trained faculty for medical students during their emergency medicine rotations were shared in order to inculcate an appreciation of the software interfaces to be used. Roles of the resident-educator were described and the modes of communication between them and the faculty-in-charge was also explained. Reading materials on debriefing tools such as the PEARLS healthcare debriefing tool, Team Strategies and Tools to Enhance Performance and Patient Safety (TeamSTEPPS) and "The Diamond" Debriefing Tool, were also shared (10-14).

\section{Writing of Simulation Scenarios}

A total of three CBS sessions were held over a 4-month period. Each session had a range of four to six resident-educator volunteers with a mix of both junior (those in the first three years of their residency training) and senior residents (those in the fourth and final years of their residency training). 
Each scenario was written by a junior resident-educator and reviewed by a senior resident-educator. All these scenarios were then reviewed by an emergency medicine residency faculty. The resident-educators used a standardised scenario writing template and it comprises of the following domains:

a. Clinical learning objectives

b. Overall case summary

c. Screen display (e.g., photo of patient, electrocardiogram [ECG], chest X-ray [CXR], lab investigations)

d. Patient state (e.g., physical examination findings, vital signs)

e. Learner's expected actions

f. Modifiers/triggers (e.g., situation for a moderator to step in)

The results of the pre-participation survey from the medical students were made available to the resident-educator before they began writing the simulation scenarios. This was so that they had these objectives in mind as they planned the scenarios for execution. The aim was also to have a mix of both ward-based scenarios as well as emergency medicine-based scenarios, so as to prepare the medical students in handling cases that they might encounter in the ward as junior doctors as well as common cases that were seen in the emergency department resuscitation area. The scenarios were pitched at the level of training of the participating medical students, whilst aiming to meet the specific learning objectives, as well as addressing the fears expressed by the students in the pre-participation survey.

At baseline, all simulation scenarios were designed to allow the medical students to demonstrate ability to conduct focused history taking, physical examination, generate a list of differential diagnoses, order and interpret relevant investigations and institute proper management for a critically-ill patient, as a team. The vetted simulation scenarios were passed on to the faculty coordinator two weeks prior to the date of the actual sessions. Further clarifications and modifications were then made as necessary. All the experiences and feedback from the preceding CBS sessions were also passed on to the resident-educator in charge of the subsequent sessions, so that the inputs could be used to help enhance the learning experience of the students. The participating medical students for each session were also provided with postparticipation feedback forms to complete so that their experience and feedback can be used to customise and improve the subsequent sessions.

In this manner, a total of three simulation sessions were conducted over four months with four simulation scenarios per session. The examples of the scenarios are listed in Table 1. These scenarios were conceptualised entirely by the residenteducators and they represent a range of acute clinical presentations.

\section{Preparing the Medical Students}

The target audience ranged from Year-1 to Year-3 medical students where the Year-1 to Year-2 were in their pre-clinical years and Year-3 would have had some form of clinical exposure. Year-1 medical students were "silent observers" and not part of the participating team. As they were part of the simulation interest group, they were very interested to observe and be a part of the learning activity, even if they did not have the hands-on practice for now. A mix of Year-2 and Year-3 medical students formed five-member teams for each scenario. The roles required of the team members were described in Table 2. Members selfallocated their own roles within the team prior to the simulation session with the Year-3 typically being chosen as the team leader. Relevant pre-reading and postreading materials were also provided for the medical students. These were sent to them for their prior reading, using a flipped classroom model. 
Table 1: The spectrum of CBS scenarios for the different sessions

\begin{tabular}{lll}
\hline First session & Second session & Third session \\
\hline $\begin{array}{l}\text { Acute shortness of breath from } \\
\text { acute pulmonary edema. }\end{array}$ & $\begin{array}{l}\text { Acute shortness of breath in a } \\
\text { post-operative patient. }\end{array}$ & Anaphylaxis. \\
$\begin{array}{l}\text { Road traffic accident with } \\
\text { pneumothorax requiring }\end{array}$ & $\begin{array}{l}\text { Exacerbation of chronic pulmonary } \\
\text { disease. }\end{array}$ & $\begin{array}{l}\text { Pulmonary embolism. } \\
\text { Sepsis shock secondion. }\end{array}$ \\
$\begin{array}{l}\text { cholangitis. } \\
\begin{array}{l}\text { Ruptured ectopic pregnancy } \\
\text { with shock. }\end{array}\end{array}$ & $\begin{array}{l}\text { Acute shortness of breath in a young } \\
\text { lady with diabetic ketoacidosis. }\end{array}$ & Intracranial hemorrhage. \\
\hline
\end{tabular}

Table 2: The different roles taken on by the medical students in each team

\begin{tabular}{ll}
\hline Roles & Description of roles \\
\hline Team leader & Responsible in leading the entire team. \\
& Communicating with patient and taking focused history. \\
& Asking for significant physical exam findings. \\
& Call for help and make arrangements for ward/ICU bed. \\
& Responsible for maintaining the patient's airway and breathing. \\
Airway doctor & Responsible for taking blood investigations, performing ultrasound, \\
Circulation doctor & $\begin{array}{l}\text { administering drugs and alternating CPR with nurse. } \\
\text { Responsible for taking the appropriate apparatus/consumables needed } \\
\text { Circulation nurse }\end{array}$ \\
& $\begin{array}{l}\text { Monitoring vitals. } \\
\text { Doing CPR. } \\
\text { Responsible for documentation, i.e., time stamps for drugs and/or any } \\
\text { other significant events in the scenario, and communicating back to team } \\
\text { members. Types in the chat box in real time in order to keep everyone } \\
\text { else updated. }\end{array}$ \\
\hline
\end{tabular}

\section{The Stages of CBS}

The stages of our CBS comprises of relatively similar steps as in manikin-based simulation. The main difference will be in the lack of the "touch and feel" and handson portions.

a. Pre-brief: Whereby the relevant instructions and information is shared, assignment of roles is done and familiarisation takes place. b. The simulation: This is where the student-centric simulation takes place. There will be tasks involved such as patient assessment, appropriate decision-making and interventions, ordering investigations and interpreting results, administration of relevant medication, reviewing the list of differential diagnoses as well as customising the appropriate interventions and management. 
c. Debrief: This is similar to manikinbased simulation but conducted virtually through the computer screens. As it is not face to face, some familiarisation and getting use to is necessary. This is also a critical phase whereby learning and reflection will take place. There is some flexibility to a debrief model which the resident-educator or faculty choose to adopt. It is also possible to have a co-debriefer and the coordination between them is important so as not to confuse the learners. During face-to-face simulation, when the learners are very young and novice in simulationbased learning, faculty may opt to use micro-debriefing with rapid cycle deliberate practice as their debriefing option. This was not used with the resident-educator group who may lack experience and moreover, with CBS, this can be a little bit more challenging. Thus, if the students were to reach a "roadblock" during the running of the simulated scenario, they may need to have prompts, which may be given as directed questions or advice (9-10).

In the pre-briefing stage, introduction of participants is important. This is followed by some of the following elements and pointers $(6-7,9)$ :

a. Stating the basic assumptions of the simulation-based training session (i.e., that every participant is assumed to be knowledgeable, is capable, and has the intention to improve/learn.

b. Explaining the principles of crisis resource management across all domains, namely situational awareness, teamwork, communications and decisionmaking.

c. Defining and clarifying the fiction contract pertaining to the "suspension of disbelief." It can be a unique challenge to act though the "scenario" is real. An engaging faculty will be able to create the fictional environment to draw participants in.

d. Reinforcing the process of learning together and from each other.

e. Maintaining confidentiality of all that go on in the session. This is also the point where facilitators and faculty will get the informed consent forms from their simulation centre signed by all participants. In our CBS, the participants provided verbal agreement to be part of the session.

f. Orientation and familiarisation with the room or simulation laboratory as well as all the equipment and layout. For CBS, this is substituted with the understanding of the processes involved in the execution of the simulation.

g. Taking and handling any queries and feedback from participants, to ally anxieties and clear doubts.

\section{The Conduct of the CBS Scenario}

Each simulation session had four scenarios and two participating teams. Each team would do two simulation scenarios during each session and team member roles would change between the different scenarios. Each scenario was estimated to last about 15 minutes with a 30-40 minutes debrief. The simulation was conducted via Zoom (a cloud-based video conferencing service) with the help of the faculty.

At the commencement of each session, the faculty would brief the participating teams regarding the netiquette of CBS e.g., while one team is actively participating, the observers will have their videos and microphones turned off. Also, the need to describe an action during a CBS clearly; e.g., "I am putting on the BP cuff for 
the patient and checking her BP now". It was emphasised that if it is not said or verbalised, the action or task would be considered as "not having been done" and the values or vitals which are demanded for will not be shown on screen. Following this, the senior resident would then address the participating teams by recapping the learning objectives for the simulation session and also ease in the participating team by emphasising the safe learning environment and nature of the simulation session. The resident-educator who wrote the simulation scenario would play the role as the simulated patient. He or she would also be the one leading the debriefing of the team at the end of the scenario. The experience from the first simulation session prompted the spontaneous creation of an additional clinical moderator role (e.g., pharmacist-on-call, advanced practice nurse). The role of the clinical moderator is to guide the medical students through their clinical reasoning process as most of the medical students were not yet familiar with the clinical management of certain critical conditions or the finer details related to pharmacology. Typically, the senior resident leading the resident-educator group would take on this role.

During the conduct of the simulation, the resident-educators will keep a keen eye on the students' level of understanding of the fundamentals such as history taking, what examination findings would be important, the investigations to order as well as initial and life-saving interventions they need to make. These would have to be verbalised clearly in CBS, unlike in the normal faceto-face simulation. These systematic steps outline their mental process and also clinical reasoning, which is core requirement to the good practice of medicine $(9,15-16)$. This must be inculcated from early on in their medical career. This is because clinical reasoning is a core and fundamental skill that they will continue to apply in practice for many years to come. Clinical reasoning involves data gathering, data interpretation and analysis, as well as making management decisions. It also involves knowledge acquisition, its application and thinking about these. Clinical reasoning is a complex process and the more these students are exposed to experiential exposures such as the CBS, the stronger these skills can be inculcated and understood (15-18).

\section{Debriefing of Medical Students}

Following the simulation, there will be the debriefing session, and this serves as another platform for active and opportunistic learning, inculcation of reflection, and, often with this, an opportunity to change behaviour or correct practice as necessary (6). It is important that debriefing can be carried out by faculty who have been trained on how to maximise learning and draw the most out of the participants, as well as instil the elements of psychological safety (19). Debriefing resident-educators must be cognizant of the participants' reaction to the simulation. Participants may react differently to the same scenario. This may be coloured by their exposure and life experiences (18-20). Psychological safety is an important precursor to learning oriented behaviour such as asking questions, sharing thoughts and making clarifications. Psychological safety will provide the students with a sense of comfort, make them feel more at ease and thus develop trust with the resident-educators more readily (19-21). The rapport and interaction between the two groups can have long lasting effect as they may work together in future. Psychological safety can at times, be even more critical than physical wellness (Table 3).

The debriefing was led by the designated senior resident upon completion of each CBS scenario. Students were asked how they felt at the end of the simulation scenario. They were reassured that their performance, emotions, sharing and other comments would be kept confidential and will only be used for the current learning activity. Even as the debriefing is conducted virtually, it was important for the facilitators 
Table 3: Characteristics of psychological safety in teams

Every team member's goals and objectives are aligned with a shared mental model (which must be known to everyone).

Ability to trust team members to assist and support each other.

Communication which is open and respectful towards each other.

Communications and actions are assumed to be carried out with good and positive intentions and thus, no "hidden agendas".

Ability to brainstorm and challenge each other's ideas in a collegial and positive manner.

Each member is accountable for his/her own actions and inputs.

An open and supportive environment to learn from mistakes and inaccurate decisions.

to realise the importance of verbal and nonverbal communications cues and promote psychological safety of the participants (9, 19-21). The resident-educators are taught to observe closely the reactions and comments from the students as they complete the "simulation". From this, they can gather whether the students are excited, relieved, disappointed or even confused. Observing their facial expressions and gestures are important in CBS. Following their comments and reaction closely will be helpful. Once the students have settled upon completion of the scenario, the learning objectives can be revisited once more to bring everyone back to the same mental model. The individual participants' views and inputs are then heard. They are also encouraged to ask question and make clarifications (6-7, 10). Resident-educators are taught to use the advocacy-inquiry line of questioning if they wish to explore certain actions carried out by the students. Using this technique, they can state: "I notice that after the airway was secured, you went on to order the drugs... Can you share with us, what exactly was going through your mind at that point in time?" With the appropriate tone, this way of enquiry sounds more palatable and less judgemental (22-24). They are free to use any of the debriefing models currently available, as long as they cover the relevant pointers in debriefing (22-23).

The resident-educators need to have an "eagle eye" to observe even the slightest nuances from the learners' perspectives.
They must be aware of these and also assist to nurture and urge participation in a neutral and non-threatening way. Once learners feel comfortable, they may then start to share and talk more openly, for example during debriefing. Being nonjudgemental is critical. Just one statement which is offensive to one learner can throw us back many steps.

In Asia, culture plays an essential role. In general, Asian learners may tend to be less vocal, less confrontational and may thus appears to be less participatory and quiet. Hierarchy play a critical role as well, whereby respect for teachers and faculty is still something still held very strongly. Thus for faculty working in cross-cultural settings, there is a need to be aware of this. Giving our learners second chances and opportunities is also important. At times a more personalised approach may even be needed for someone who is extremely shy and fearful of "public speaking".

At the conclusion of the debriefing segment, the resident-educators will go through the scenario to reinforce the learning points for the students. This is an important customisation as this was the first foray for the students into the world of simulation and CBS (24-26).

\section{Debriefing of Resident-Educator}

At the end of the simulation session, the faculty would give a live debrief to the resident-educators. This is similar to 
"debriefing the debriefer". How they felt during each stage would be shared openly and addressed $(22-23,25,27)$. This was also where new ideas were generated and changes may then be considered for incorporation into the next CBS session. The faculty doing the debriefing would seek the resident-educators' inputs on how they felt the scenario was executed, whether it was too difficult, too easy or just right. Their views will be substantiated with examples from the session. Their reaction to the students' performance is also important to extract, as their focus on both verbal and non-verbal communications can be ascertained. This is also where they reflect on all aspects of the CBS, step by step. In particular, as this is a virtually conducted learning session and relatively new to everyone, their inputs pertaining to this was also polled. It was heartening to note that they found it a refreshing change to the usual methodologies of teaching and were excited and enthusiastic to plan the next session. The sharing also enabled these resident-educators to be more aware of their capabilities, mind-set and their collaboration with their colleagues.

After this debrief, they were also provided with the delayed feedback from the students obtained through an online postparticipation survey. This can thus be used for the resident-educators learning portfolio as they continue to develop their teaching capabilities and repertoire.

\section{RESULTS}

From this pilot project on CBS conducted by the resident-educators, there were many observations made. A total of six resident educators were involved in a training with a total of 72 of Year-1 to Year-3 medical students, over a period of four months. Both junior and senior residents were able to be a part of and contributed to the project. Faculty oversight was useful; there was support from the medical school as well as clinical support from a Faculty of Emergency Physician.
Preparation and practice before conducting the actual CBS was essential. Writing suitable scenarios, putting all the clinical information together (including radiological images, blood results, photographs, electrocardiograms), practice of running the scenarios and coordination of roles represented all the back-room tasks to be completed before these CBS can be run smoothly. The prior survey was useful to gather needs and expectations. Across all the three groups, their choice of the top three learning objectives from the pre-determined list provided as well as their own inputs were:

a. Gaining greater exposure to emergency case management;

b. To strengthen their clinical reasoning; and

c. To learn about team work and teambased management.

As for their top three fears or concerns, these were:

a. The concern about their own incompetence;

b. Worry that they may cause harm to patients, due to the incompetence perceived; and

c. Concerns on too much pressure or stress.

These theme appeared to be recurring across the three batches of students. The observations and results were shared and discussed with the resident-educator prior to the conduct of the CBS so that they could pay particular attention to address these and also reinforce the psychological safety to the students.

The other observation made was that virtual debriefing requires a lot of patience and can be more time consuming. The debriefers have to observe facial expressions and body language through the computer screens. There must be a room for more questions to clarify certain intentions, when not 
clear. Virtual debriefing must also be paced appropriately, because if too fast, students may not be able to get the message being shared adequately. Noting also the learners in this case include medical students in their early years of study. The resident-educators enjoyed the session tremendously as they managed to learn as they taught the students and even fine-tune their own clinical acumen. The final comment from students, resident-educators and the facilitators was that CBS such as this is very useful but will be even better when used as prior training before the actual face-to-face simulation of these scenarios are conducted. COVID-19 gave the resident-educators an impetus to create this CBS platform to create a prior learning activity, whilst awaiting a suitable time when the actual simulation laboratory face-to-face sessions can be executed.

\section{DISCUSSION}

CBS represents the use of a computer to imitate a real world process, scenario or system. There are indeed several benefits of CBS. It is logistically easy to put together without violating the social distancing rules during the COVID-19 pandemic. During our CBS, Year-1 medical students get exposure to simulation via silent observation of Year-2 and Year-3 medical students' participation. The familiarisation process should not be under-estimated. It is a safe virtual learning environment for all. In fact, these CBS sessions can potentially be expanded to include trainee nurses to help promote inter-professional education $(9,28)$. Debriefing sessions just for these observer groups to address their questions and learning needs should also be included in the planning of future CBS sessions (9).

In CBS, there is active interaction between the interphase and the participants. This can be in the form or audio, visual or touch capabilities. For example, there could be the use of mouse clicks, touch screens or voice prompts. Simulation can help to contextualise theoretical medical knowledge into actual practical applications. In CBS, the participants face their computer screens all the time - hence, the role-playing by the resident-educators and the beeping sound of the vital signs monitors are the two key elements that can help to make the ambience feel as if the students are in the actual resuscitation room, despite the fact that they are actually online (9). It was also an interesting observation that the behaviour of the medical students participants was influenced by the beeping sound of the vital signs monitor - the faster the beeping went with the tachycardia from the "patient" in the scenario, the more pressurised the students sounded (as evidenced by their verbal and non-verbal communications being observed virtually), with more prompt actions undertaken to correct the patient's worsening physiology. Likewise, the more realistic the acting by the resident-educators (e.g., grabbing the chest in severe pain, shouting in pain) and the more pressurised the participants appeared to be, the higher the tendency for them to miss certain steps in the clinical reasoning process to expeditiously address the current issue on hand.

Simulation-based teaching has been extensively utilised in many medical schools and residency programmes, with favourable outcomes $(1,6-8,18,24)$. Teaching these same medical concepts over cloud-based video conferencing service such as Zoom, is however much less studied (9). There were some limitations, for instance, it was difficult to teach skills such as how to "bag and mask" or intubate a patient. There are certainly limitations to the actual "touch and feel" procedures and skills. However, focus can be shifted to understanding clinical concepts such as that of rapid sequence induction and working through the different algorithmic steps of Advanced Cardiac Life Support modules. In addition, step-by-step clinical reasoning processes, teamwork with close-loop communications and listening to inputs from other members of the group are also important (15). Due to the interface challenge with CBS, it was 
difficult for the participants to simulate simultaneous actions (9). For example, putting on the vital signs monitor while interviewing the patient and examining the patient cannot happen all at the same time. Even procedures have to be verbalised and cannot proceed simultaneously. Also, closeloop communication has to happen in order for the task to be done.

As near-peer educators, residents are close enough to the medical students they teach to understand the challenges they face and help plan the optimal approach for them to learn effectively (5). This collaboration is particularly interesting for the residenteducators as designing and conducting simulation training is not formally being taught as a part of the curriculum of the residency programme. It has to be taken as a separate course or as an educational training module as a faculty. While the residents have routinely been playing the participant roles in real life simulations designed by the residency faculty, this is the first time for many of the residenteducators to experience CBS and to be on the "other side" of the simulation scenario. Participation in these teaching and mentorship opportunities is purely on a voluntary basis, at the expense of the residents' own time. For this collaboration, most resident-teachers only have the twohour introduction to CBS before they began writing the simulation scenarios. The main challenge faced by the residenteducators were setting the difficulty level of the scenarios appropriate for the medical students. In a few of the scenarios, the level of difficulty of the simulation scenarios were pitched a little too high, and hence, requiring the creation of the clinical moderator role as they went along.

Hence, there is a need for the presence of a residency faculty member who is experienced in running simulation to be present and guide the resident-educators to achieve the learning objectives. As the resident-educators' role are a longitudinal and ongoing one, these residents will be supported with the relevant formal instructions, support and mentoring to enhance their teaching skills.

\section{CONCLUSION}

In these unprecedented times of COVID-19 pandemic, finding alternative ways to enhance and continue medical education is necessary. CBS is an innovative way to continue medical student education despite the current limitations and distancing restrictions. It has the potential to reach a wider learner group and perhaps promote inter-professional education. With the distraction of hands-on component taken off, it allows a shift in focus to clinical concepts, clinical reasoning and soft skills such as close-loop team communications. Resident-educators should receive formal training with faculty supervision in designing simulation scenarios to be executed using CBS format.

\section{ACKNOWLEDGEMENTS}

The team would like to acknowledge the following persons for their assistance in running the CBS sessions; Kirsty Freeman and Abegail Resus Fernandez (Duke-NUS Medical School, Clinical Performance), Hoe Pei Shan and the Duke-NUS Emed SIG.

\section{REFERENCES}

1. Ten ERP, Tews M, Ballester JM. Improved medical student satisfaction and test performance with a simulationbased emergency medicine curriculum: a randomized controlled trial. Ann Emerg Med. 2009;54(5):684-91. https://doi.org/10 $.1016 /$ j.annemergmed.2009.03.025

2. Goh SH, Tan HWJ, Cook S. Students' perspective of residents as educators following introduction of ACGME-I in SingHealth residency. Proceedings of Singapore Healthcare. 2016;25(3):169-75. https://doi.org/10.1177/2010105816641971 
3. Mann K, Sutton E, Frank B. 12 tips for preparing residents as teachers. Med Teach. 2007;29:301-6. https://doi.org/10.1080/ 01421590701477431

4. Morrison E, Shapiro J, Harthill M. Resident doctors' understanding of their roles as clinical teachers. Med Educ. 2005;39:13744. https://doi.org/10.1111/j.1365-2929 .2004.02063.x

5. Bulte C Betts A, Garner K, Durning S. Student teaching: views of students near peer teacher and learner. Med Teach. 2007;29(6):583-90. https://doi.org/10.1080/ 01421590701583824

6. Lateef F. Simulation based learning: just like the real thing. J Emerg Trauma Shock. 2010;3(4):348-52. https://doi.org/10.4103/ 0974-2700.70743

7. Pothiawala $S$, Lateef F. Simulation training in emergency medicine: an important component of residency curriculum. Hong Kong Journal of Emergency Medicine. 2012;19:41-5. https://doi.org/10 $.1177 / 102490791201900107$

8. Bond WF, Spillane L. The use of simulation for emergency medicine resident assessment. Acad Emerg Med. 2002;9(11):1295-9. https://doi.org/10.1197/aemj.9.11.1295

9. Lateef F. Computer-based simulation and online teaching netiquette in the time of COVID-19. EC Emergency Medicine and Critical Care. 2020;4(8):84-91.

10. Eppich W, Cheng A. Promoting excellence and reflective learning in simulation (PEARLS): development and rationale for a blended approach to healthcare simulation debriefing. Simul Healthc. 2015;10(2):106-15. https://doi.org/10.1097/ SIH.0000000000000072

11. Bajaj K, Meguerdichian M, Thoma B. Eppich W, Cheng A. The PEARLS healthcare debriefing tool. Acad Med. 2017;93(2):336. https://doi.org/10.1097/ ACM.0000000000002035
12. Castner J. Validity and reliability of the brief TeamSTEPPS teamwork perceptions questionnaire. J Nurs Meas. 2012;20(3):186-98. https://doi.org/10.1891/ 1061-3749.20.3.186

13. Lineberry M, Bryan E, Brush T, Carolan T, Holness D, Salas E, King H. Measurement and training of TeamSTEPPS dimensions using the medical team performance assessment tool. Jt Comm J Qual Patient Saf. 2013;39(2):89-95. https://doi.org/10 $.1016 /$ S1553-7250(13)39013-8

14. Jaye P, Thomas L, Reedy G. The Diamond: a model for simulation debrief. The Clin Teach. 2015;12(3):171-5. https://doi.org/10 $.1111 /$ tct. 12300

15. Lateef F. Clinical reasoning: the core of medical education and practice. International Journal of Internal and Emergency Medicine. 2018;1(2):1-7.

16. Schmidt HG, Mamede S. How to improve the teaching of clinical reasoning: a narrative review and proposal. Med Educ. 2015;49(10):961-73. https://doi.org/10 $.1111 /$ medu. 12775

17. Post RE, Quattlebaum G, Benich J. Residents-as-teachers curricula: a critical review. Acad Med. 2009;84(3):374-80. https://doi.org/10.1097/ACM.0b013e318 $1971 \mathrm{ffe}$

18. Snell L. The resident-as-teacher: it's more than just about student learning. J of Grad Med Educ. 2011;3(3):440-1. https://doi.org/ 10.4300/JGME-D-11-00148.1

19. Lateef F. Maximising learning and creativity: understanding psychological safety in simulation-based learning. J Emerg Trauma Shock. 2020;13:5-14.

20. Edmondson AC, Lei Z. Psychological safety: the history, renaissance and future of inter-personal construct. Ann Rev Organ Psychiatry Organ Behav. 2014;1:23-43. https://doi.org/10.1146/annurev-orgpsych $-031413-091305$ 
21. Rudolph JW, Raemer DB, Simon R. Establishing a safe container for learning in simulation: the role of the pre-simulation briefing. Simul Healthc. 2014;9:339-49. https://doi.org/10.1097/SIH .0000000000000047

22. Kolbe M, Grande B, Spahn DR. Briefing and debriefing during simulation-based training and beyond. Content, structure, attitude and setting. Best Pract Res Clin Anaesthesiol. 2015;29:87-96. https://doi.org/ 10.1016/j.bpa.2015.01.002

23. Rudolph JW, Simon R, Dufresne R, Raemer DB. There is no such thing as "non-judgemental" debriefing: a theory and method for debriefing with good judgement. Simul Healthc. 2006;1(1):49-55. https://doi .org/10.1097/01266021-200600110-00006

24. Reedy GB. Using cognitive load theory to plan simulation design and practice. Clin Sim Nurs. 2015;11(8):355-60. https://doi .org/10.1016/j.ecns.2015.05.004
25. Lateef F. The art of conscious practice of medicine: mastering medicine. Education in Medicine Journal. 2016;8(2):83-7. https://doi.org/10.5959/eimj.v8i2.416

26. Cook DA, Triola MN. Virtual patients: a critical literature review and the next steps. Med Educ. 2009;43(4):303-11. https://doi .org/10.1111/j.1365-2923.2008.03286.x

27. Savery JR. Be VOCAL: characteristics of successful online instructors. Journal of Interactive Online Learning. 2005;4(2):14152.

28. Lateef F. Inter-professional education and inter-professional practice and team science: learning together; working together. Education in Medicine Journal. 2018;10(4):81-91. https://doi.org/10.21315/ eimj2018.10.4.8 